\title{
PROLONGED EXTERNAL PRESSURE AS A CAUSE OF ISCHAEMIC CONTRACTURE
}

\author{
Alan N. Conner, Glasgow, Scotland \\ From the Western Infirmary, Glasgow
}

Ischaemic contracture of the forearm is usually caused by occlusion of the brachial artery (Griffiths 1940) or by increased tension in a closed fascial compartment following a crush injury (Parkes 1959). Local ischaemic damage to the thenar and hypothenar muscles may be produced by tight bandaging (Parkes 1966).

Howse and Seddon (1966) drew attention to the occurrence of muscle necrosis in association with carbon monoxide poisoning, overdoses of alcohol or hypnotics, and even general anaesthesia, and reported four patients with carbon monoxide or barbiturate poisoning in whom external pressure of an amount considered insufficient to damage normal tissues had caused necrosis of the underlying muscles of the forearm and hand. This particular cause of ischaemic contracture has not been widely recognised, but with the increasing incidence of barbiturate poisoning there will be many more patients at risk. A further six cases are therefore reported and the clinical features, diagnosis and management discussed.

\section{CASE REPORTS}

Case 1-A woman aged eighteen was given a combination of morphine, atropine and adrenaline for severe bronchospasm and thereafter slept on her right side for fifteen hours. When she awoke there were signs of prolonged pressure on the skin of her right cheek, shoulder and forearm. Three days later the volar surface of the forearm was swollen and tender. There was complete loss of function of the median and ulnar nerves in the forearm and hand and a partial lesion of the posterior interosseous nerve. Immediate decompression of the flexor compartment of the forearm was carried out. The bellies of flexor carpi radialis and flexor digitorum superficialis appeared dead but the main trunks of the median and ulnar nerves were normal. Although there was transient induration of the affected muscle bellies gradual recovery took place, and a year later she had almost normal function in the hand.

Case 2-A forty-five-year-old man with a history of alcoholism was admitted to hospital, having taken a large quantity of alcohol and butobarbitone. He had been unconscious for twelve hours and appeared to have superficial burns of his left side involving the face, shoulder, hip, forearm and hand. These healed quickly but he was referred to the hand clinic eighteen months later with an adduction contracture of the left thumb. There was no sensory loss. The affected thenar muscles were excised in an attempt to overcome the contractures but he has not attended since discharge. From further enquiries it was clear that he could not have been burnt, and it was more likely that the "burn" and underlying muscle necrosis had been caused by direct pressure from lying on his left side.

Case 3-A thirty-six-year-old man with a history of alcoholism was admitted to hospital, having taken an overdose of pethidine, butobarbitone and alcohol. He had been unconscious for twelve hours. There were areas of erythema and blistering on the right side of the face and chest and on the front of the right forearm and hand which were thought to be superficial burns. The serum barbiturate level was 3.3 milligrams per 100 millilitres and the $\mathrm{pO}_{2}$ was 48 millimetres $\mathrm{Hg}$. Treatment by forced diuresis and oxygen therapy rapidly improved the level of consciousness. Five days later, because of a high fever and brawny swelling of the 
forearm and hand, a palmar space infection was suspected but no pus was found on incision. The local signs settled over the next two weeks and he was discharged from hospital.

He was seen at the hand clinic six weeks later because of residual stiffness of the fingers, and was found to have an adduction contracture of the thumb, contractures of the intrinsics of the index and middle fingers and a flexion contracture of the wrist. The thenar muscles, intrinsics and long flexors were indurated. There was loss of sensation to light touch in the distribution of the median and ulnar nerves but a delayed response to pain. Careful questioning established that he could not have been burnt but that he had been found lying on his right side.

Six weeks later the flexor compartment of the forearm was explored. There was an area of muscle necrosis affecting the greater part of the radial head of flexor digitorum superficialis and more deeply the bellies of flexor pollicis longus and flexor digitorum profundus to the index finger. A segment of the median nerve about three centimetres long at the level of the mid-forearm was also ischaemic. The affected part of flexor digitorum superficialis was excised. The adherent slips of flexor pollicis longus and flexor digitorum profundus were freed and the belly of flexor pollicis longus was lengthened in a " $z$ " fashion at the site of the scarring. The flexor tendons to the wrist were also lengthened. Microscopy of the excised muscle showed aggregates of necrotic muscle fibres. At the periphery of the necrotic areas the muscle tubes contained macrophages and there was extensive fibrous replacement of the muscle bundles. Further improvement was gained three months later by excision of all the contracted muscles of the thenar eminence and a Littler release of the index, middle and ring fingers. A year after the injury he had regained useful function in the hand, but he was not able to return to his previous employment as an asphalt spreader.

Case 4-A fifty-two-year-old woman was admitted to hospital with what were thought to be superficial burns on the front of the left forearm and the left thenar eminence. She had a previous history of depression and drug overdosage, and on this occasion had taken a large quantity of barbiturates and had been unconscious for thirty-six hours. She was referred to the hand clinic six months later because of persistent stiffness of the fingers. From a review of the history, it was apparent that she could not have burnt the limb. She had spent the period while unconscious lying on the floor of her home although her exact position is unknown. She had the typical signs of ischaemic contracture affecting the thenar muscles, the intrinsics in the first intermetacarpal space and the long flexors. She appreciated light touch in the distribution of the median and ulnar nerves but stated that this had only recovered in the previous six weeks.

Tenotomy of the wrist flexors and excision of the collateral ligaments to the index and middle fingers allowed the wrist to come to the neutral position and improved the range of flexion at the metacarpo-phalangeal joints. The flexor compartment of the forearm was explored and there was a large area of muscle necrosis involving the bellies of flexors digitorum superficialis and profundus. These were separated from one another and from the interosseous membrane. Intensive physiotherapy gave some improvement but a year after the injury the fingers are still flexed. She has reasonable use of the hand, good sensation, and can do all her own housework.

Case 5-A forty-five-year-old woman was admitted to hospital with what appeared to be superficial burns of the left hand, there being swelling, erythema and blistering over the thenar eminence and dorsum of the first and second metacarpals. Two days later she was thought to have a thenar space infection which was treated with antibiotics. She presented at the hand clinic two weeks later with an adduction contracture of the thumb. The skin was healed but the thenar muscles and first dorsal interosseous were wasted and fibrosed. The history revealed that she had lain unconscious for one and a half hours following an epileptiform seizure and had been clear of any fires. On regaining consciousness she noticed that her left hand was painful and swollen. She denied the possibility of drug overdosage although she had

VOL. 53 B, NO. 1, FEBRUARY 1971 
previously taken an overdose of barbiturates. Three months after the injury the fibrosed muscles were excised and she has regained good function in the hand.

Case 6-A forty-seven-year-old lorry driver was admitted in a confused, delirious state following an influenzal-like illness and was sedated on several occasions with chlorpromazine 200 milligrams and intramuscular hyoscine 0.4 milligram. During this time, after sleeping for four hours on his right side, he was noted to have signs of pressure on the skin of his right cheek, chest and forearm, and within forty-eight hours the forearm was swollen and tender.

There were no contractures, but any attempt to straighten the flexed fingers was painful. There was complete paralysis of the extensors of the fingers and thumb and flexor digitorum superficialis. Flexor pollicis longus, flexor digitorum profundus, and the small muscles of the hand were also affected, contracting to power 3 . There was no sensation in the distribution of the median, ulnar or superficial radial nerves. In an attempt to improve the circulation in the muscle bellies, a tourniquet was applied to the forearm at a pressure midway between the systolic and diastolic blood pressures (Parkes 1966), but after four hours there was no improvement in sensation. The following day there was slight power in all the paralysed muscles and three months later all the muscles were contracting to power 4 . There has been no recovery of sensation and he has not been able to return to his employment. The precise cause of the acute delirium remains obscure; it was possibly caused by hyponatraemia secondary to viral hepatitis.

\section{DISCUSSION}

Howse and Seddon (1966) concluded that these lesions are caused by a combination of hypoxacmia and local external pressure. Their four patients had been unconscious from coal gas or barbiturate poisoning. Linton, Adams and Lawson (1968) reported a case of ischaemic contracture of the calf muscles following coal gas poisoning, the patient's legs having lain across a fender. In all these cases it was suggested that systemic hypoxia had predisposed to

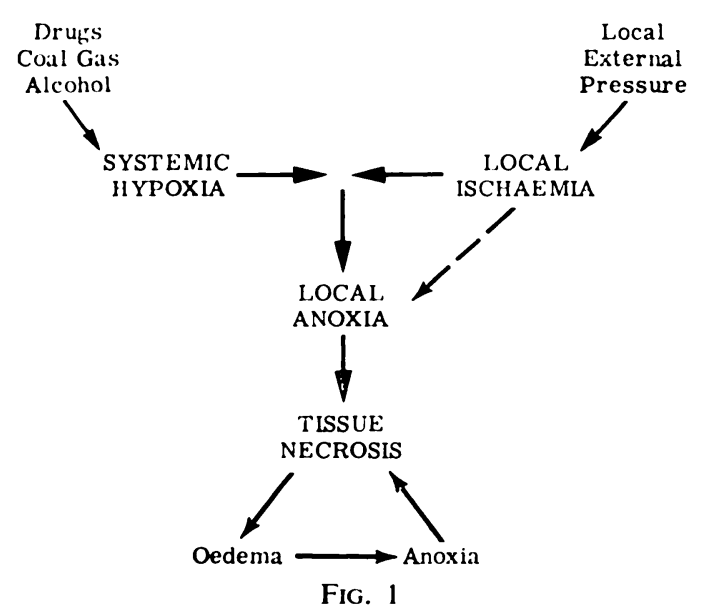

The pathogenesis of tissue necrosis following prolonged external pressure. a discrete area of muscle death when the tissues were subjected to external pressure of a degree which would not normally be expected to cause necrosis.

In the present series one patient had no drugs and two others only pharmacological doses of central nervous system depressants. This suggests that external pressure alone may be able to cause muscle necrosis provided it is continuous and prolonged. Parkes (1966) noted the occurrence of ischaemic contracture of the intrinsic muscles of the hands by their compression between the buttock and operating table during a long operation under general anaesthesia. Systemic hypoxaemia, from the causes mentioned, cannot of course be ignored and may reduce the amount of pressure required to cause tissue death at a given site. The most important effect of carbon monoxide or barbiturate poisoning, however, is probably unconsciousness; it thus provides the environment for prolonged local pressure. Once the process starts the breakdown of muscle fibres in a closed fascial compartment causes swelling with further anoxia and tissue death (Fig. 1).

Histologically the lesions rescmble Volkmann's ischaemic contracture but differ macroscopically in that the affected muscles are related to the site of pressure and not to the area of tissue supplied by an artery. The nerve damage is also ischaemic, but although muscle 
necrosis is visible within a few days, naked eye evidence of nerve involvement is not apparent for several weeks.

There are three recognisable clinical stages: skin necrosis, muscle necrosis and residual deformity. The first, which lasts about seventy-two hours, is characterised by discrete areas of erythema and blistering which mimic superficial burns. These are usually multiple and occur in seven common sites (Fig. 2), which are related to the position in which the patient has been lying. In the cases described by Howse and Seddon (1966) the lesions were restricted to the forearm and hand.

The second stage lasts for two weeks, and with the exception of the case described by Linton et al. (1968) has been confined to the upper limb. The volar surface of the forearm or the thenar eminence is swollen, red and tender and may be mistaken for a fascial space infection (Cases 3 and 5). In only two patients was the true nature of the underlying pathology recognised at this stage, but careful examination should reveal evidence of nerve involvement.

Finally, as the necrotic muscle is replaced by fibrous tissue, fixed deformities develop. The muscles commonly affected are flexor carpi radialis, flexors digitorum superficialis and profundus and flexor pollicis longus in the forearm, and the thenar muscles and first dorsal interosseous in the hand.

The diagnosis requires an awareness of the condition and a careful history and examination, often difficult in the early stages. The patient may

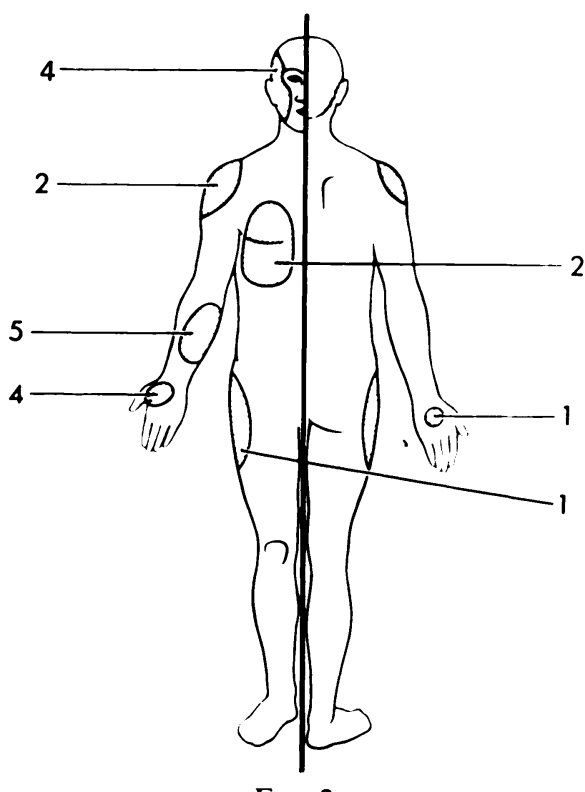

FIG. 2

The distribution of "burns" in six patients with ischaemic contractures of the forearm or hand. have a history of drug overdosage. There has usually been a prolonged period of unconsciousness during which the patient has lain undisturbed, and, although the skin lesions look like burns, their distribution, the absence of marks on the clothing, of fires near the patient, and the presence of nerve involvement should suggest the correct diagnosis.

Treatment depends on the stage of the disease. Initially, when swelling in a tight fascial compartment is suspected, the overlying deep fascia should be widely split and the resulting skin defect covered with a split-skin graft. This treatment appears to have been successful in Case 1. It seems logical to follow this with high-pressure oxygen therapy which has the added advantage of providing some protection against anaerobic infection of the ischaemic muscles.

While preparations are being made to split the deep fascia it is worth while trying the effect of applying a pneumatic tourniquet to the forearm as described by Parkes (1959). The cuff, applied over the swollen muscles, is inflated to about 70 millimetres $\mathrm{Hg}$., thus encouraging circulation in the tense muscle compartment. After half an hour nerve function in the limb is carefully assessed: if there is no improvement surgery should not be further delayed. All these measures should be combined with elevation of the affected limb and an intensive programme of active and passive joint movement.

By the time most patients are seen, however, there are fixed deformities which cannot be corrected by physiotherapy. Muscles in which there is no remaining healthy tissue should be excised: this is usually successful in mobilising an adducted thumb. Simple division or lengthening of wrist flexor tendons will correct the deformity at the wrist but a wide dissection of the forearm is required to correct flexion contractures of the fingers. The contracted muscle bellies should be freed from one another and from the underlying interosseous membrane and those which are completely fibrosed should be excised. Often the fibrous tissue is confined 
to a segment in the centre of the muscle, and this may be lengthened in a " $z$ " fashion. Metacarpo-phalangeal joints which are stiff in extension may be mobilised by excision of the collateral ligaments, and severe intrinsic contractures with flexed metacarpo-phalangeal joints by excision of the affected muscles.

The nerve lesions have a good prognosis and require no more than a neurolysis.

\section{SUMMARY}

1. Six patients are reported in whom ischaemic muscle contractures followed prolonged external pressure.

2. Systemic hypoxaemia may be a predisposing factor in patients with overdoses of central nervous system depressants.

3. If treatment is to be successful, early diagnosis is required, and the most important factor is an awareness of the condition.

4. The treatment of the late case is discussed.

I am grateful to Mr A. R. Parkes for permission to report these patients under his care and for his helpful criticism and advice in the preparation of this paper. The illustrations were kindly prepared by the Department of Medical Illustration, Western Infirmary, Glasgow.

\section{REFERENCES}

Griffiths, D. L. (1940): Volkmann`s Ischaemic Contracture. British Journal of Surgery, 28, 239.

Howse, A. J. G., and Seddon, Sir H. (1966): Ischaemic Contracture of Muscle Associated with Carbon Monoxide and Barbiturate Poisoning. British Medical Journal, 1, 192.

Linton, A. L., Adams, J. H., and Lawson, D. H. (1968): Muscle Necrosis and Acute Renal Failure in Carbon Monoxide Poisoning. Postgraduate Medical Journal, 44, 338.

Parkes, A. R. (1959): Treatment of Traumatic Tension Ischaemia of Muscle and Nerve. Journal of Bone and Joint Surgery, 41-B, 628.

Parkes, A. R. (1966): Ischaemic Contracture. Clinical Surgery. Edited by Charles Rob and Rodney Smith -7 The Hand, edited by R. G. Pulvertaft, p. 257. London: Butterworths. 